\title{
Streda Formula for the Hofstadter-Wilson-Dirac Model in Two and Four Dimensions
}

\author{
Takahiro Fukui and Takanori Fujiwara \\ Department of Physics, Ibaraki University, Mito 310-8512, Japan
}

(Dated: July 27, 2018)

\begin{abstract}
We rederive the spectral asymmetry of the Wilson-Dirac model in external fields, paying attention to the Chern number due to the Berry connection. We interpret the smooth part of the spectral asymmetry as the Streda formula that is originally derived for the two-dimensional quantum Hall effect (QHE). We show by numerical calculations that the Streda formula reproduces the known first and second Chern numbers in a weak magnetic field limit. We conjecture that the Streda formula is valid even for stronger fields and for more generic systems in higher dimensions.
\end{abstract}

\section{INTRODUCTION}

Some symmetries in classical systems are broken in corresponding quantized systems $\stackrel{1-4}{-4}$ These phenomena are called anomalies in field theories, and have played an important role in particle physics. The chiral anomaly successfully explained the color degrees of freedom of quarks before the advent of QCD, and the anomaly cancellation $^{5,6}$ provides a powerful guideline in unified gauge theories. It is well known that they are closely related with the topological structures of underlying gauge theories as summarized in the index theorem. Topological aspects of anomalies imply their universality in physics. Indeed, the $\mathrm{QHE}$ can be interpreted ${ }^{7}$ as the parity anomaly of the Dirac fermion in three space-time dimensions. $\stackrel{8}{-}$ According to the development of topological insulators, there is a growing interest in various kinds of anomalies in condensed matter physics, 9,10 Recently, topological phases in gapless systems, called Weyl and Dirac semimetals, have been proposed, $11-13$ and observed experimentally: ${ }^{14,15}$ This motivates us to reconsider the experimental observations of the chiral anomaly of Weyl fermions ${ }^{16-19}$ in a crystal proposed long ago. ${ }^{20}$

In this paper, we investigate the Wilson-Dirac model in strong external fields, which is referred to as Hofstadter-Wilson-Dirac (HWD) model, defined on the Euclidean two- and four-dimensional spaces. One of the purposes of this paper is to show an intimate relationship between the chiral anomaly of the Dirac fermion in particle physics and the topological insulating phase in condensed matter physics. Indeed, Qi et al. have studied the same model to construct the theory of the topological insulator $\stackrel{9}{-}$ In Sect. II A. we briefly introduce the recent development of lattice gauge theory concerning the chiral anomaly on the lattice. Here, the spectral asymmetry plays a key role. In Sect. IIB. we then rederive $e^{9.21}$ the smooth part of the spectral asymmetry, paying attention to how the Chern number due to the Berry curvature appears. We find that the relationship between the spectral asymmetry and the chiral anomaly is quite similar to the Streda formula, ${ }^{22}$ which represents the Hall conductivity (and thus the first Chern number) as the number of occupied states in the QHE. Thus, the chiral anomaly represented by the spectral asymmetry that we discuss in this paper is also referred to as the
Streda formula. Remarkably, the Streda formula enables us to compute the Chern number using eigenvalues only, without using eigenstates. It turns out that the Streda formula is valid even in four dimensions, and it serves as an efficient tool for computing the second Chern number related with the chiral anomaly. In Sects. III and IV. we numerically show that the Streda formula reproduces the known Chern numbers of the HWD model in the weak field limit in not only two dimensions but also four dimensions. We finally conjecture in Sect. V V that the Streda formula holds more generic systems even in four dimensions, and numerically suggest second Chern numbers for several Landau levels formed in a simple tight-binding model.

\section{HOFSTADTER-WILSON-DIRAC MODEL}

The Hamiltonian in $d$-dimensions is defined as

$$
\begin{aligned}
H= & \frac{-i t}{2} \sum_{\mu=1}^{d} \sum_{j}\left(e^{-i \phi_{\mu, j}} c_{j}^{\dagger} \gamma_{\mu} c_{j+\hat{\mu}}-\text { h.c. }\right)+m \sum_{j} c_{j}^{\dagger} \gamma_{d+1} c_{j} \\
& +\frac{b}{2} \sum_{\mu=1}^{d} \sum_{j}\left(e^{-i \phi_{\mu, j}} c_{j}^{\dagger} \gamma_{d+1} c_{j+\hat{\mu}}+\text { h.c. }-2 c_{j}^{\dagger} \gamma_{d+1} c_{j}\right) \\
\equiv & \sum_{i, j} c_{i}^{\dagger} \mathcal{H}_{i j} c_{j}
\end{aligned}
$$

where $\hat{\mu}$ is the unit vector in the $\mu$-th $(\mu=1, \cdots, d)$ direction and the $\gamma$-matrices mean for $d=2$ the Pauli matrices $\gamma_{1}=\sigma_{1}, \gamma_{2}=\sigma_{2}, \gamma_{3}=\sigma_{3}$, while they mean for $d=4$ the standard hermitian $4 \times 4 \gamma$-matrices. The phase $\phi_{\mu, j}$ stand for an external gauge field, which will be specified momentarily. We investigate the spectral flow as a function of a uniform magnetic field, i.e., the Hofstadter butterfly. Here, the spectral asymmetry plays a key role in extracting the topological nature from the butterfly, which is defined as

$$
\eta \equiv \frac{1}{2} \operatorname{Tr} \frac{\mathcal{H}}{\sqrt{\mathcal{H}^{2}}}=\frac{N_{+}-N_{-}}{2},
$$

where Tr implies the trace over the $\gamma$-matrices as well as the space $j$, and $N_{ \pm}$stands for the numbers of the positive and negative energy states of $\mathcal{H}$. 


\section{A. Chiral anomaly on the lattice}

The spectral asymmetry (2) is closely related with chiral symmetry on the lattice: The naive chiral symmetry is broken by the Wilson term even when $m=0$ in Eq. (1). Without the Wilson term, the fermion suffers from the doubling and the chiral anomaly cancels out among the fermion and the doublers. $\frac{23,24}{2}$ It is known, however, that we can define the chiral invariant lattice fermion using the Dirac operator $D$ satisfying the GinspargWilson relation $25 \gamma_{d+1} D+D \gamma_{d+1}=a D \gamma_{d+1} D$, where $a$ is the lattice constant. This leads to chiral symmetry on the lattice: $D \gamma_{d+1}(1-a D / 2)+(1-a D / 2) \gamma_{d+1} D=$ $0{ }^{26}$ The chiral anomaly is then given by ${ }^{26-28} \mathcal{A}_{x}=$ $\operatorname{tr} \gamma_{d+1}\left(1-\frac{a}{2} D\right)_{x, x}$, where $x=a j$ stands for the lattice coordinates and tr implies the trace over the $\gamma$-matrices. Such $D$ can be explicitly found as the overlap Dirac operator $^{29,30} D=\frac{1}{a}\left(1-\gamma_{d+1} \frac{\mathcal{H}}{\sqrt{\mathcal{H}^{2}}}\right)$. The spectral asymmetry (2) can be related with the chiral anomaly by the lattice version of the index theorem $\eta=\sum_{x} \mathcal{A}_{x} \cdot \underline{31}$ Thus, $\eta$ is topological.

\section{B. Continuum limit of the spectral asymmetry}

If a state flows across zero energy, $\eta$ changes discontinuously. Therefore, $\eta$ is composed of the smooth part $\bar{\eta}$ and the discontinuous part $\eta_{\mathrm{d}}$,

$$
\eta=\bar{\eta}+\eta_{\mathrm{d}}
$$

Below, we derive $\bar{\eta}$, according to Ref ${ }^{21}$, especially paying attention to how the Chern number associated with the Berry curvature appears. To this end, we calculate $\eta$ up to $a^{d}$. Let $\nabla$ and $\nabla^{*}$ be the forward and backward difference operators, respectively, defined as

$$
\begin{aligned}
& a \nabla_{\mu} c_{x}=e^{a A_{\mu}(x)} c_{x+a \hat{\mu}}-c_{x}, \\
& a \nabla_{\mu}^{*} c_{x}=c_{x}-e^{-a A_{\mu}(x-a \hat{\mu})} c_{x-a \hat{\mu}},
\end{aligned}
$$

where $A_{\mu}(x) \equiv-i \phi_{\mu, j} / a$ is purely imaginary. The fermion operators have been labeled by $x=a j$. Note that $\sum_{x} c_{x}^{\dagger}\left(a \nabla_{\mu} c_{x}\right)=\sum_{x, y} c_{x}^{\dagger}\left(a \nabla_{\mu} \delta_{x, y}\right) c_{y}$, where $a \nabla_{\mu}$ in the r.h.s operates on $x$ of $\delta_{x, y}$. Then, as the first quantized form, $\mathcal{H}$ in Eq. (1) is denoted as

$$
\mathcal{H}_{x, y}=\hat{\mathcal{H}} \delta_{x, y} \equiv \gamma_{I} \hat{X}_{I} \delta_{x, y},
$$

where $\hat{X}_{I}$ operates to $x$ and is defined as

$$
\hat{X}_{I}= \begin{cases}\frac{-i t}{2} a\left(\nabla_{\mu}+\nabla_{\mu}^{*}\right) & (I=\mu) \\ m+\frac{b}{2} \sum_{\mu=1}^{d} a\left(\nabla_{\mu}-\nabla_{\mu}^{*}\right) & (I=d+1)\end{cases}
$$

Noting $\delta_{x, y}=\int_{-\pi}^{\pi} e^{i \frac{k}{a} \cdot(x-y)} \frac{d^{d} k}{(2 \pi)^{d}}$, we have the representation of $\eta$ suited for deriving the continuum limit,

$$
\begin{aligned}
\eta & =\frac{1}{2} \sum_{x} \operatorname{tr}\left(\frac{\mathcal{H}}{\sqrt{\mathcal{H}^{2}}}\right)_{x, x} \\
& =\frac{1}{2} \sum_{x} \int_{-\pi}^{\pi} e^{-i \frac{k}{a} \cdot x} \operatorname{tr} \frac{\hat{\mathcal{H}}}{\sqrt{\hat{\mathcal{H}}^{2}}} e^{i \frac{k}{a} \cdot x} \frac{d^{d} k}{(2 \pi)^{d}} .
\end{aligned}
$$

Next, let us calculate the $\eta$ in the small $a$ limit. Note that

$$
\begin{aligned}
e^{-i \frac{k}{a} \cdot x} a \nabla_{\mu} e^{i \frac{k}{a} \cdot x} & =e^{i k_{\mu}} a \nabla_{\mu}+e^{i k_{\mu}}-1 \\
& =i K_{\mu}+\delta_{\nu} K_{\mu} a D_{\nu}+O\left(a^{2}\right),
\end{aligned}
$$

where $D_{\mu}$ is the covariant derivative defined as

$$
D_{\mu}=\partial_{\mu}+A_{\mu}
$$

with $\partial_{\mu}=\partial / \partial x_{\mu}$, and $K_{\mu}$ is an exponentiated momentum $k_{\mu}$ defined as $i K_{\mu} \equiv e^{i k_{\mu}}-1$. The derivative with respect to $k_{\mu}$ is denoted as $\delta_{\mu}$ such that $\delta_{\nu} K_{\mu} \equiv$ $\partial K_{\mu} / \partial k_{\nu}=\delta_{\mu \nu} e^{i k_{\mu}}$ to distinguish it from $\partial_{\mu}$. Likewise, we have

$$
e^{-i \frac{k}{a} \cdot x} a \nabla_{\mu}^{*} e^{i \frac{k}{a} \cdot x}=i K_{\mu}^{*}+\delta_{\nu} K_{\mu}^{*} a D_{\nu}+O\left(a^{2}\right) .
$$

Using Eqs. (6) and (7), we derive $\hat{\mathcal{H}}$ up to order $a$ below. First, $\hat{X}_{I}$ in Eq. (4) is linear in $\nabla_{\mu}$ and $\nabla_{\mu}^{*}$, so that

$$
e^{-i \frac{k}{a} \cdot x} \hat{X}_{I} e^{i \frac{k}{a} \cdot x}=X_{I}-i \delta_{\mu} X_{I} a D_{\mu}+O\left(a^{2}\right)
$$

where $X_{I}=X_{I}(k)$ is calculated as $X_{\mu}=(-i t / 2) i\left(K_{\mu}+\right.$ $\left.K_{\mu}^{*}\right)=t \sin k_{\mu}(\mu=1, \cdots, d)$ and $X_{d+1}=m+$ $(b / 2) \sum_{\mu} i\left(K_{\mu}-K_{\mu}^{*}\right)=m+b \sum_{\mu}\left(\cos k_{\mu}-1\right)$. Then, we readily have

$$
\begin{aligned}
e^{-i \frac{k}{a} \cdot x} \hat{\mathcal{H}} e^{i \frac{k}{a} \cdot x} & =\gamma_{I} X_{I}-i \gamma_{I} \delta_{\mu} X_{I} a D_{\mu}+O\left(a^{2}\right), \\
e^{-i \frac{k}{a} \cdot x} \hat{\mathcal{H}}^{2} e^{i \frac{k}{a} \cdot x} & =X^{2}+\hat{O}-\gamma_{I J} \delta_{\mu} X_{I} \delta_{\nu} X_{J} a^{2} F_{\mu \nu}+O\left(a^{3}\right),
\end{aligned}
$$

where we have defined $X^{2}=X_{I}^{2}, \gamma_{I J}=\left[\gamma_{I}, \gamma_{J}\right] / 4, \hat{O}=$ $-2 i X_{I} \delta_{\mu} X_{I} a D_{\mu}-\delta_{\mu} X_{I} \delta_{\nu} X_{I} a D_{\mu} a D_{\nu}$, and

$$
F_{\mu \nu}(x)=\left[D_{\mu}, D_{\nu}\right]=\partial_{\mu} A_{\nu}-\partial_{\nu} A_{\mu}
$$

is the field strength of the external fields.

The smooth part $\bar{\eta}$ in Eq. (5) is the contribution of order $a^{d}$ : We compute the following in the limit $a \rightarrow 0$ : 


$$
\frac{\bar{\eta}}{a^{d}}=\frac{1}{2 a^{d}} \sum_{x} \int_{-\pi}^{\pi} \frac{d^{d} k}{(2 \pi)^{d}} \operatorname{tr} \frac{\gamma_{I} X_{I}-i \gamma_{I} \delta_{\mu} X_{I} a D_{\mu}}{\sqrt{X^{2}+\hat{O}-\gamma_{I J} \delta_{\mu} X_{I} \delta_{\nu} X_{J} a^{2} F_{\mu \nu}}} .
$$

Using the series expansion $\frac{1}{\sqrt{1-x}}=\sum_{n=0}^{\infty} \frac{(2 n-1) ! !}{n ! 2^{n}} x^{n}$ and $\operatorname{tr} \gamma_{I_{1}} \gamma_{I_{2}} \cdots \gamma_{I_{d+1}}=(2 i)^{d / 2} \epsilon_{I_{1} I_{2} \cdots I_{d+1}}$, we have

$$
\begin{aligned}
\frac{\bar{\eta}}{a^{d}} & =\frac{1}{2} \sum_{x} \int_{-\pi}^{\pi} \frac{d^{d} k}{(2 \pi)^{d}} \frac{(2 n-1) ! !}{n ! 2^{n} X^{2 n+1}} \operatorname{tr} \gamma_{I} X_{I}\left(\gamma_{I J} \delta_{\mu} X_{I} \delta_{\nu} X_{J} F_{\mu \nu}\right)^{n}+O(a) \\
& =(-1)^{n+1} c_{n} \frac{i^{n}}{n !(2 \pi)^{n} 2^{n}} \sum_{x} \epsilon_{\mu_{1} \nu_{1} \cdots \mu_{n} \nu_{n}} F_{\mu_{1} \nu_{1}} \cdots F_{\mu_{n} \nu_{n}},
\end{aligned}
$$

where we have set $d=2 n$ to consider even $d$, and

$$
c_{n} \equiv \frac{(2 n-1) ! !(-1)^{n+1}}{2(2 \pi)^{n}} \int_{-\pi}^{\pi} d^{d} k \frac{\epsilon_{I I_{1} \cdots I_{d}}}{X^{2 n+1}} X_{I} \delta_{1} X_{I_{1}} \cdots \delta_{d} X_{I_{d}}
$$

is the $n$-th Chern number. Equation (8) has been derived in Ref $\underline{\underline{9}}$.

\section{Chern number associated with Berry connection}

We show that Eq. (9) is the Chern number associated with the many-body ground state. The Chern number is defined as

$$
c_{n}=\frac{1}{n !}\left(\frac{i}{2 \pi}\right)^{n} \int \operatorname{tr} f^{n}
$$

where $f$ is the Berry curvature 2 -form $f=\delta a+a^{2}$ defined using the Berry connection 1-form $a=\psi^{\dagger} \delta \psi$. Here, $\psi$ is the multiplet wavefunction of the negative energy states and $\delta$ is the external derivative with respect to $k_{\mu}: \delta g=$ $\left(\partial g / \partial k_{\mu}\right) d k_{\mu}=\delta_{\mu} g d k_{\mu}$. It is known that

$$
\operatorname{tr} f^{n}=\operatorname{tr}\left(P(\delta P)^{2}\right)^{n}=\operatorname{tr} P(\delta P)^{2 n},
$$

where $P$ is the projection operator to the ground state, $P=(1-\mathcal{H} / X) / 2$ with $\mathcal{H}(k)=\gamma_{I} X_{I}(k)$. Then,

$$
\begin{aligned}
\operatorname{tr} f^{n} & =\frac{-1}{(2 X)^{2 n+1}} \operatorname{tr} \gamma_{I} X_{I} \gamma_{I_{1}} \delta X_{I_{1}} \cdots \gamma_{I_{d}} \delta X_{I_{d}} \\
& =\frac{-(2 i)^{n}(2 n) ! d^{d} k}{(2 X)^{2 n+1}} \epsilon_{I I_{1} \cdots I_{d}} X_{I} \delta_{1} X_{I_{1}} \cdots \delta_{d} X_{I_{d}} .
\end{aligned}
$$

Substituting this into Eq. (10), we arrive at Eq. (9).

\section{A strategy for strong fields}

Thus far, we have derived $\eta$ in the $a \rightarrow 0$ limit, which also implies the weak field limit. Indeed, $c_{n}$ in Eq. (10) is given by $\psi$ or $P$ in the zero field limit. However, as shown by Lüscher, $\stackrel{32}{=} \eta$ on the lattice is generically given by the form of Eq. (8), and thus, we expect that the effects of finite $a$ and strong fields simply renormalize $c_{n}$ and $\eta_{\mathrm{d}}$. Here, the topological nature of $\eta$ should constrain $c_{n}$ to be an integer. In what follows, we regard $c_{n}$ as an unknown integer and determine it by the numerical computation of $\eta$.

\section{TWO-DIMENSIONAL HWD SYSTEM}

Let us consider a finite system under the periodic boundary condition in a uniform magnetic field $B$. We assume a commensurate magnetic flux per plaquette,

$$
B a^{2} \equiv \phi=\frac{2 \pi p}{q}, \quad p=0, \cdots, q,
$$

and consider the spectral flow as a function of $p$ with a fixed $q$. This is the famous Hofstadter butterfly. We
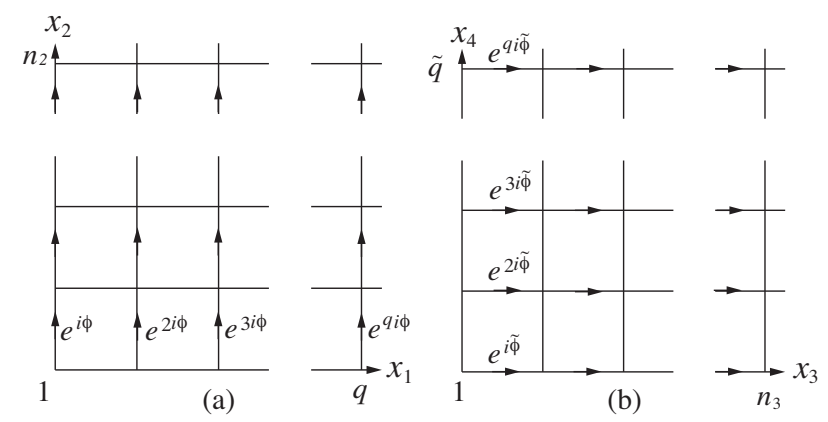

FIG. 1: Landau gauge for a magnetic field (a) and an electric field (b). The system sizes toward $x_{1}$ and $x_{4}$ are $q$ and $\tilde{q}$, whereas they are $n_{2}$ and $n_{3}$ toward $x_{2}$ and $x_{3}$, respectively. The periodic boundary condition is imposed.

take the Landau gauge as depicted in Fig. 1(a). The 
Hofstadter butterfly is shown in Fig. 2(a) for the model whose ground state has $c_{1}=1$ when $\phi=0$. Several other Chern numbers computed directly using the Berry curvature ${ }^{33-35}$ are also shown.
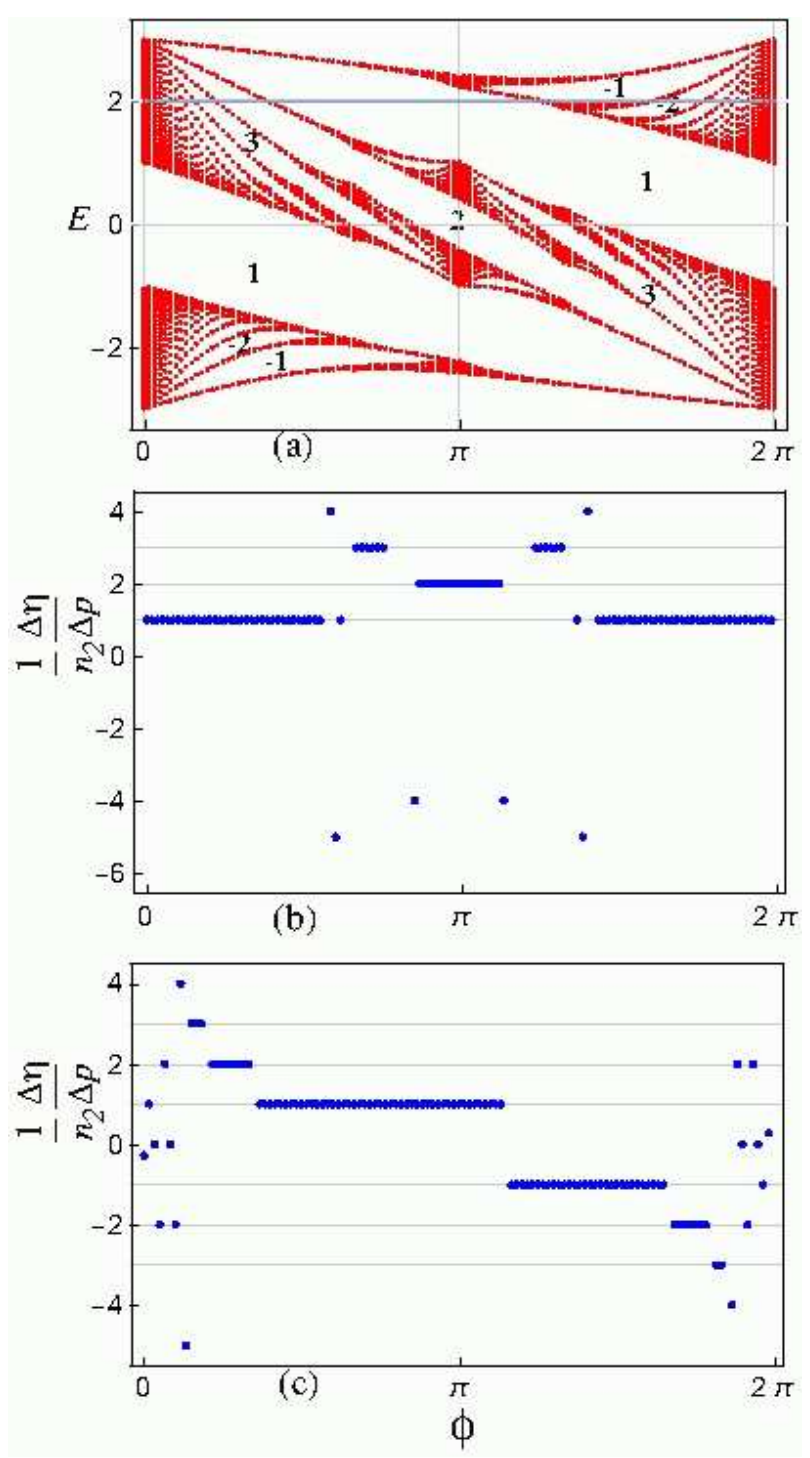

FIG. 2: HWD model in $d=2$. The parameters used are $t=1, m=1, b=1$, and $q=120$. The system size in $x_{2}$ is $n_{2}=120$. (a) is the spectrum as a function of the magnetic flux per plaquette, $\phi$. Several Chern numbers for the occupied states below the gap are indicated. (b) and (c) are computed using the Streda formula $(\Delta \eta / \Delta p) / n_{2}$ in Eq. (14) at zero energy and at the energy $\mu=2$, respectively.

$\bar{\eta}$ is given by (8) with $i F_{12}=B$ : We find

$$
\bar{\eta}=\frac{c_{1}}{2 \pi} \sum_{x} B a^{2}=\frac{c_{1}}{2 \pi} B a^{2} \cdot q n_{2}=c_{1} p n_{2}
$$

where $q n_{2}$ is the number of the plaquettes on the plane.
Substituting this into Eq. (3), we reach

$$
\eta=\frac{c_{1}}{2 \pi} B a^{2} \cdot q n_{2}+\eta_{\mathrm{d}}=c_{1} p n_{2}+\eta_{\mathrm{d}} .
$$

To see that this leads to the Streda formula, we note that the density of states below zero energy is $n_{-} \equiv$ $N_{-} /\left(q n_{2} a^{2}\right)$. We also note that $\eta_{\mathrm{d}}$ is a function of $B$, but it should be constant if the energy gap is open at zero energy: When the gap closes and spectral flow occurs across zero energy, it discontinuously changes by an integer. From Eq. (2), we have $\eta=N / 2-N_{-}$, where $N$ is the total number of states. Thus, we reach

$$
\left.\frac{d n_{-}}{d B}\right|_{\text {smooth }}=-\frac{c_{1}}{2 \pi} .
$$

This corresponds to the Streda formula for the WilsonDirac model. For the practical numerical computations, it is convenient to regard $\eta$ as a function of $p$ with $q$ fixed. Then, Eq. (12) is converted into

$$
\frac{1}{n_{2}} \frac{\Delta \eta}{\Delta p} \equiv \frac{\eta(p+1)-\eta(p)}{n_{2}}=c_{1}+\Delta \eta_{\mathrm{d}}^{\prime},
$$

where $\Delta \eta_{\mathrm{d}}^{\prime} \equiv \Delta \eta_{\mathrm{d}} / n_{2}$ is obviously zero when the Fermi energy is in the bulk gap.

In Fig. 2(b), we show the 1.h.s of Eq. (14) computed directly from the numerical results of $N_{ \pm}$in (a). We see several flat regions when the zero energy is in the bulk gap. From the r.h.s, it turns out that these are just the first Chern numbers of the ground state. Indeed, they are consistent with the Chern numbers indicated in Fig. 2(a). On the other hand, if the gap closes, Eq. (14) rapidly jumps. This is due to $\Delta \eta_{\mathrm{d}}^{\prime}$.

For reference, we compute the model including the chemical potential $-\mu \sum_{j} c_{j}^{\dagger} c_{j}$ in the Hamiltonian. In this case, $\eta$ can be computed by redefining $N_{ \pm}$as the numbers of states above and below the energy $\mu$. We show the result in Fig. 2(c). It is consistent with the Chern numbers indicated in (a). The Streda formula Eq. (13) or (14) seems valid for not only zero energy but also finite energies, as it should be, since the original Streda formula 22 is quite generic.

\section{FOUR DIMENSIONAL HWD SYSTEM}

In addition to a uniform magnetic field $B$ in Fig. 1(a), we introduce a uniform electric field $E$ in the $x_{3}$ direction, as depicted in Fig. 11(b). Note that $x_{4}$ is the imaginary time, and thus, the present Wilson-Dirac model is defined in the Euclidean space in order for the Hamiltonian to be hermitian. We assume a commensurate electric field per plaquette,

$$
E a^{2} \equiv \tilde{\phi}=\frac{2 \pi \tilde{p}}{\tilde{q}}, \quad \tilde{p}=0,1, \cdots
$$

as well as the magnetic field $\phi$ as in Eq. (11), and consider the spectral flow as a function of magnetic field. 

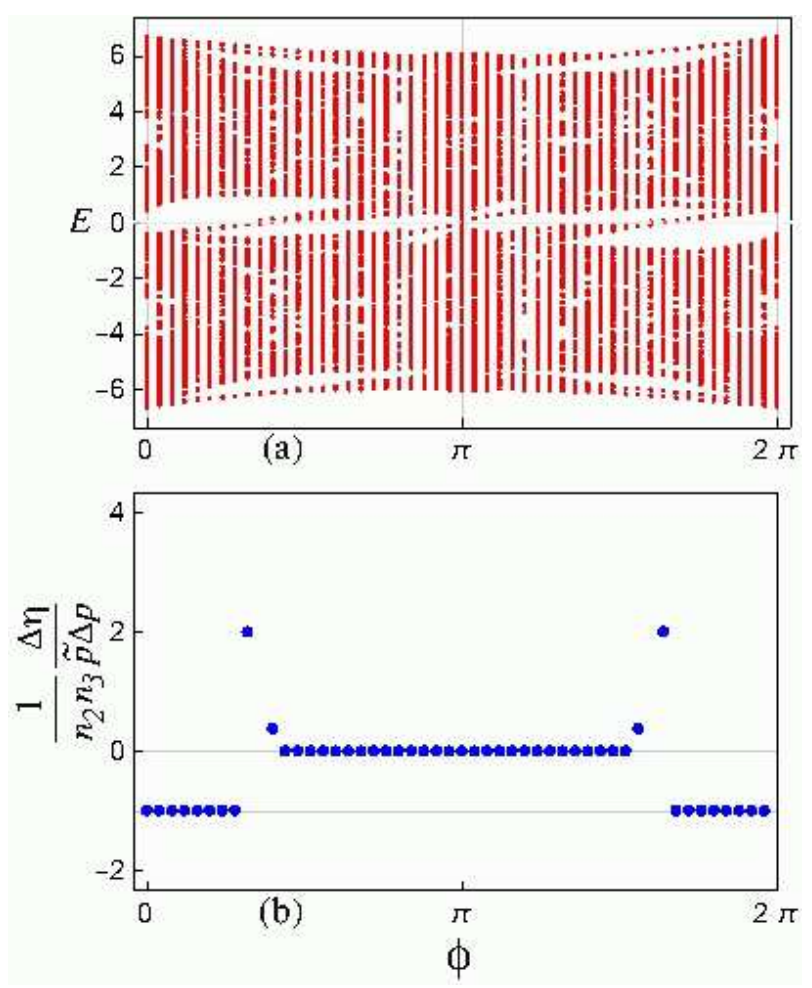

FIG. 3: HWD model in $d=4$. The parameters used are $t=1, m=1, b=1, q=50$, and $\tilde{q}=6$. The numbers of sites along $x_{2}$ and $x_{3}$ are $n_{2}=n_{3}=4$. (a) is the spectrum as a function of the magnetic flux $\phi$ for $\tilde{\phi}=\pi / 3(\tilde{p}=1)$ fixed. (b) is the Streda formula in Eq. (17).

First, let us derive the Streda formula for the fourdimensional HWD model. Setting $i F_{12}=B$ and $i F_{34}=$ $E$ in Eq. (8), we have

$$
\bar{\eta}=-\frac{c_{2}}{(2 \pi)^{2}} \sum_{x} B E a^{4}=-\frac{c_{2}}{(2 \pi)^{2}} B E a^{4} \cdot q n_{2} n_{3} \tilde{q} .
$$

This is nothing but the chiral anomaly term $\propto \mathbf{B} \cdot \mathbf{E}$. Together with Eqs. (11) and (15), we reach

$$
\eta=-c_{2} p \tilde{p} n_{2} n_{3}+\eta_{\mathrm{d}}
$$

The density of state below zero energy is given by $n_{-} \equiv$ $N_{-} /\left(a^{4} q n_{2} n_{3} \tilde{q}\right)$, so that we have

$$
\left.\frac{\partial n_{-}}{\partial(B E)}\right|_{\text {smooth }}=\frac{c_{2}}{(2 \pi)^{2}}
$$

This can be regarded as the Streda formula in four dimensions describing the relationship between the density of states and the topological invariant $c_{2}$. For the numerical calculations, as a function of $p$ and $\tilde{p}$ with $q$ and $\tilde{q}$ fixed, $\eta(p \tilde{p})$, we have

$$
\frac{1}{n_{2} n_{3}} \frac{\Delta \eta}{\Delta(p \tilde{p})}=\frac{1}{n_{2} n_{3}} \frac{1}{\tilde{p}} \frac{\Delta \eta}{\Delta p}=-c_{2}+\Delta \eta_{\mathrm{d}}^{\prime}
$$
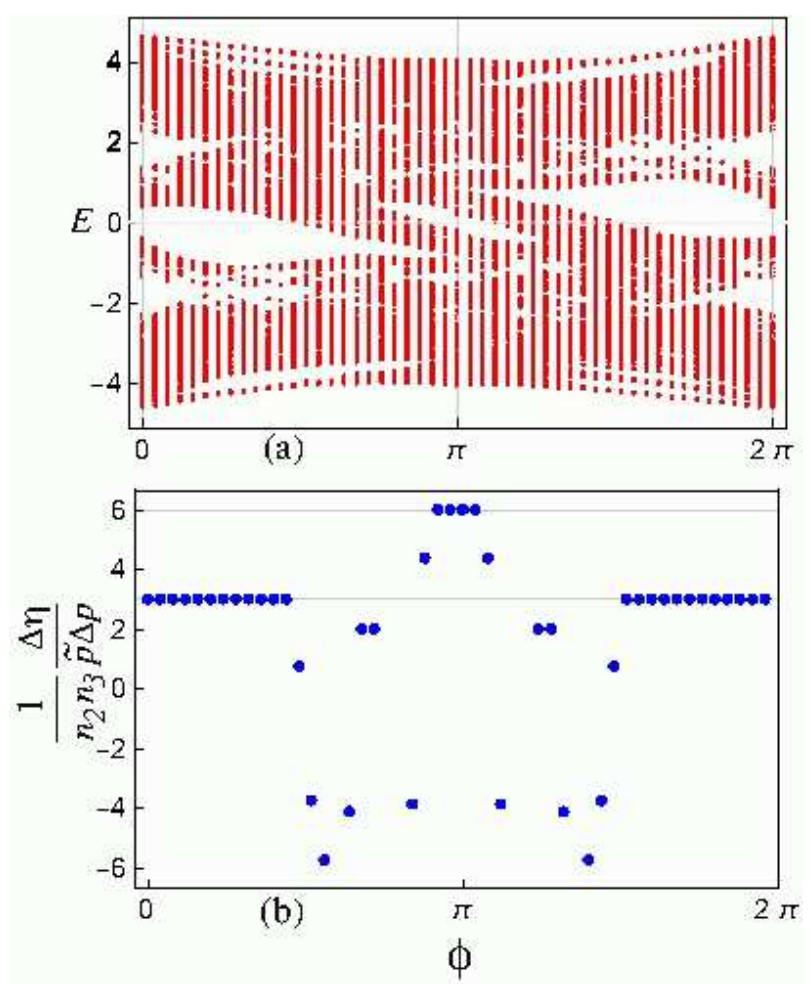

FIG. 4: HWD model in $d=4$. The parameters used are the same as in Fig. 3 except $m=3.1$. (a) is the spectrum. (b) is the Streda formula.

where the first equality means that we compute the difference of $\eta$ with respect to $p$ with $\tilde{p}$ fixed. Other notations are similar to those in Eq. (14).

In Figs. 3(a) and 4(a), we show the spectra of the HWD model as functions of the magnetic flux $\phi$ with a small electric flux $\tilde{\phi}$ fixed. Near $\phi \sim 0$, the gaps at zero energy in 3(a) and 4(a) open, which are known to have $c_{2}=1$ and -3 , respectively, at $\phi=0 \stackrel{9,21}{\underline{n}}$ We expect that even with a magnetic field, the gaps keep the same Chern numbers until the gaps close in a strong magnetic field regime. To see this, we show the numerical calculations of the l.h.s of Eq. (17) in Figs. 3(b) and 4(b). Indeed, there appear flat regions with the same Chern numbers. Therefore, we expect that the Streda formula (16) or (17) is valid even in a strong magnetic field. Interestingly, we find another gapped ground state with $c_{2}=-6$ around a very strong magnetic field $\phi=\pi$ in Fig. 四(b).

\section{GENERIC SYSTEMS: A CONJECTURE}

What we have learned in the QHE is that a simple tight-binding model, which is topologically trivial, becomes nontrivial once a magnetic field is switched on and a single band spectrum separates into many Landau levels with finite Chern numbers. This motivates us to investigate a simple tight-binding model in four di- 

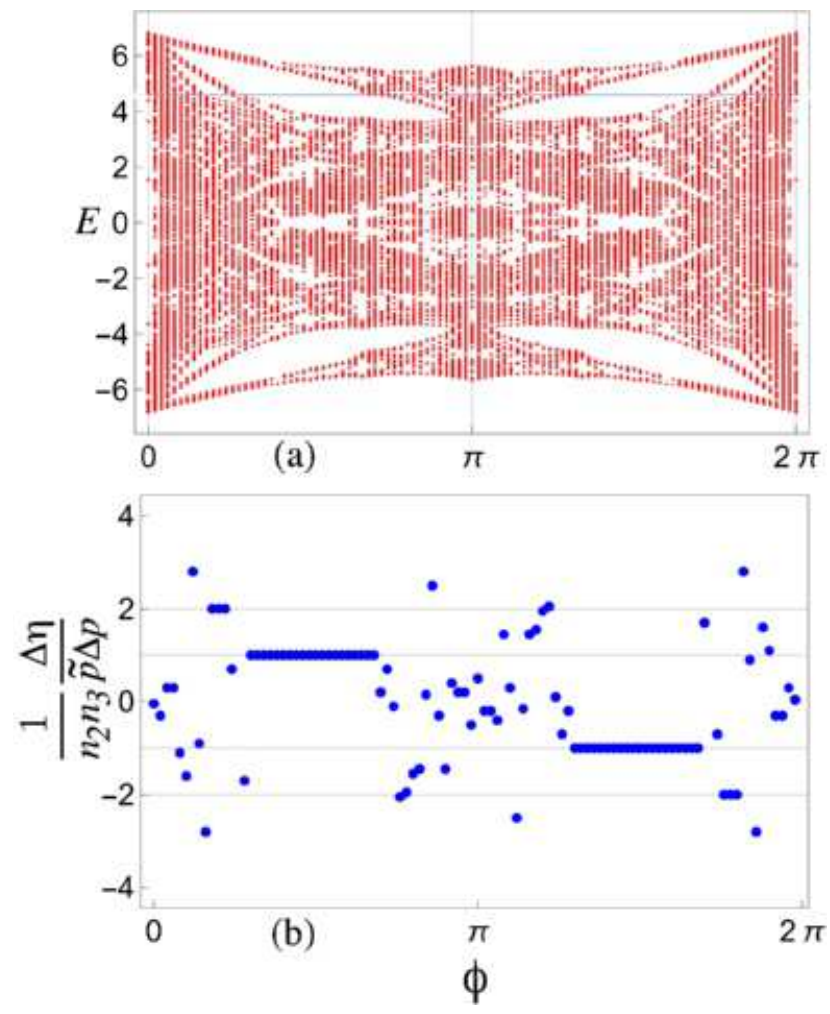

FIG. 5: A tight-binding model in $d=4$. The parameters used are $t_{\mu}=1$ (uniform hopping), $q=100$, and $\tilde{q}=16$. The system size is $n_{2}=n_{3}=10$. (a) is the spectrum for $\tilde{\phi}=\pi / 2(\tilde{p}=4)$ fixed. (b) is the Streda formula in Eq. (17) with chemical potential $\mu=4.6$. For reference, we show a horizontal thin line at energy $\mu=4.6$ in (a).

mensions,

$$
H=-\sum_{\mu=1}^{4} t_{\mu} \sum_{j}\left(e^{-i \phi_{\mu, j}} c_{j}^{\dagger} c_{j+\hat{\mu}}+\text { h.c. }\right),
$$

where the magnetic and electric fields are introduced as in Fig. 1. Here, remember that the Streda formula in Eq. (13) or (14) is derived for the HWD model in $d=2$, but it holds more generically in a model-independent manner, as shown by Streda. $\stackrel{22}{2}$ Thus, we assume that the Streda formula in Eq. (16) or (17) is also valid for more generic models in $d=4$, and apply it to the simple tight-binding model in Eq. (18).

We show in Fig. (5) (a) the spectrum of the model (18) as a function of the magnetic flux. Although the spectrum is gapless at zero energy, we find several gap structures at $E \sim \pm 5$. Let us compute the Streda formula Eq. (17)

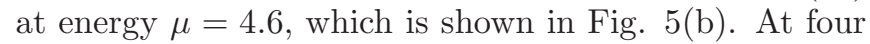
gaps from $\phi=0$ to $2 \pi, c_{2}$ reads $-2,-1,1,2$.

One may wonder if there is room for the first Chern number to characterize the numerically observed flat regions in Fig. [5. since at least in the case $t_{3}, t_{4} \ll t_{1}, t_{2}$, the model can be only a layered square lattice system. Let us consider the extreme case, $t_{3}=t_{4}=0$. In this case, the Landau levels should be characterized by $c_{1}$, since the system is copies of independent two-dimensional systems, and Landau levels obviously carry the first Chern numbers, not the second Chern numbers. Note that the number of copies is $n_{3} \tilde{q}$. Thus, Eq. (12) is modified as $\eta=c_{1} p n_{2} \cdot n_{3} \tilde{q}+\eta_{\mathrm{d}}$. Therefore, we expect in this case,

$$
\frac{1}{n_{2} n_{3}} \frac{1}{\tilde{q}} \frac{\Delta \eta}{\Delta p}=c_{1}+\Delta \eta_{\mathrm{d}}^{\prime}
$$

The differences in $\tilde{p}$ and $\tilde{q}$ in the denominators of the l.h.s in Eqs. (17) and (19) should be noted. If we interpret the observed values of the flat regions in Fig. [5 to be $c_{1}$ as the result of the two-dimensionality, Eq. (19) tells that $c_{1}=-c_{2} \cdot \tilde{p} / \tilde{q}=-c_{2} / 4=1 / 2,1 / 4,-1 / 4,-1 / 2$ from $\phi=0$ to $2 \pi$. Thus, these cannot be the first Chern numbers. If we reduce the values of $t_{3}$ and $t_{4}$, say, up to the order of $t_{1} / 10, t_{2} / 10$, the spectrum almost reproduces the two-dimensional butterfly of the square lattice system, and the first Chern numbers computed using Eq. (19) are consistent with those of the square lattice system. Therefore, it is quite natural to conclude that the uniform tight-binding model in four dimensions has a nontrivial gapped ground state characterized by $c_{2}$.

\section{SUMMARY AND DISCUSSION}

We have explored the chiral anomaly of the WilsonDirac model on the lattice in strong external fields. Taking the weak field limit for the spectral asymmetry, we have rederived the chiral anomaly, paying special attention to the Chern number due to the Berry connection. In two dimensions, the relationship between the spectral asymmetry and the chiral anomaly is the same as the Streda formula. Thus, the generalized Streda formula we have derived in this paper enables us to compute the Chern numbers using eigenvalues only, without using eigenvectors. The results of several numerical calculations have suggested that the generalized Streda formula is valid for not only the Wilson-Dirac model but also the simple tight-binding model.

It is natural that the Landau level of a system in $2 n$ dimensions allows the $n$th Chern numbers. However, there is room for lower Chern numbers, reflecting the fact that a $2 n$-dimensional system can be a layered system in lower dimensions, at least in some limit, as we have mentioned in Sect. VI The dimensionality and the order of the Chern number may be an interesting future issue. The direct derivation of the Streda formula in higher dimensions, especially in four dimensions for more generic systems, should also be addressed, since the second Chern number is relevant to a recent interesting topic, i.e., the observation of the chiral anomaly in a crystal. 


\section{Acknowledgments}

This work was supported in part by Grants-in-Aid for Scientific Research Numbers 25400388, 26247064, and
24540247 from the Japan Society for the Promotion of Science.
1 S. L. Adler, Physical Review 177, 2426 (1969), URL http://link.aps.org/doi/10.1103/PhysRev.177.2426.

2 J. S. Bell and R. Jackiw, Il Nuovo Cimento A (1965-1970) 60, 47 (1969), URL http://dx.doi.org/10.1007/BF02823296.

3 S. L. Adler and W. A. Bardeen, Physical Review 182, $1517 \quad$ (1969), URL http://link.aps.org/doi/10.1103/PhysRev.182.1517.

4 W. A. Bardeen, Physical Review 184, 1848 (1969), URL http://link.aps.org/doi/10.1103/PhysRev.184.1848.

5 C. Bouchiat, J. Iliopoulos, and P. Meyer, Physics Letters B 38, 519 (1972), URL http://www.sciencedirect.com/science/article/pii/0 6 D. J. Gross and R. Jackiw, Physical Review D 6, 477 (1972), URL http://link.aps.org/doi/10.1103/PhysRevD.6.477

7 K. Ishikawa, Physical Review D 31, 1432 (1985), URL http://link.aps.org/doi/10.1103/PhysRevD.31.1432.

8 A. N. Redlich, Physical Review Letters 52 (1984), URL http://link.aps.org/doi/10.1103/PhysRevLett.52.18.

9 X.-L. Qi, T. L. Hughes, and S.-C. Zhang, Physical Review B 78, 195424 (2008), URL http://link.aps.org/doi/10.1103/PhysRevB.78.195424

10 S. Ryu, J. E. Moore, and A. W. W. Ludwig, Physical Review B 85, 045104 (2012), URL http://link.aps.org/doi/10.1103/PhysRevB.85.045104

11 S. Murakami, New Journal of Physics 9, 356 (2007), URL http://stacks.iop.org/1367-2630/9/i=9/a=356

12 A. Burkov and L. Balents, Phys. Rev. Lett. 107, $127205 \quad$ (2011), 1105.5138, URL http://arXiv.org/abs/1105.5138

13 S. M. Young, S. Zaheer, J. C. Y. Teo, C. L. Kane, E. J. Mele, and A. M. Rappe, Physical Review Letters 108, 140405 (2012), URL http://link.aps.org/doi/10.1103/PhysRevLett.108.140405

14 Z. Wang, Y. Sun, X.-Q. Chen, C. Franchini, G. Xu, H. Weng, X. Dai, and Z. Fang, Physical Review B 85, 195320 (2012), URL http://link.aps.org/doi/10.1103/PhysRevB.85.195320

15 S.-Y. Xu, I. Belopolski, N. Alidoust, M. Neupane, G. Bian, C. Zhang, R. Sankar, G. Chang, Z. Yuan, C.-C. Lee, et al., Science 349, 613 (2015), URL http://science.sciencemag.org/content/349/6248/613.a

16 D. T. Son and B. Z. Spivak, Physical Review B 88, 104412 (2013), URL http://link.aps.org/doi/10.1103/PhysRevB.88.104412

17 S. Roy, M. Kolodrubetz, J. E. Moore, and A. G. Grushin, arXiv:1605.08445.

18 Y. Ominato and M. Koshino, Physi- cal Review B 93, $245304 \quad$ (2016), URL http://link.aps.org/doi/10.1103/PhysRevB.93.245304.

19 R. D. dos Reis, M. O. Ajeesh, N. Kumar, F. Arnold, C. Shekhar, M. Naumann, M. Schmidt, M. Nicklas, and E. Hassinger, New Journal of Physics 18, 085006 (2016), URL http://stacks.iop.org/1367-2630/18/i=8/a=085006

20 H. B. Nielsen and M. Ninomiya, Physics Letters B 130, $389 \quad$ (1983), URL http://www.sciencedirect.com/science/article/pii/03702693839

21 T. Fujiwara, K. Nagao, and H. Suzuki, Journal of High Energy Physics 2002, 025 (2002), URL

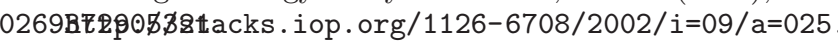
${ }^{22}$ P. Streda, Journal of Physics C: Solid State Physics 15, L717 (1982), URL http://stacks.iop.org/0022-3719/15/i=22/a=005

23 H. B. Nielsen and M. Ninomiya, Nuclear Physics B 185, 20 (1981), URL http://www.sciencedirect.com/science/article/pii/05503213819

$24 \mathrm{H}$. B. Nielsen and M. Ninomiya, Nuclear Physics B 193, 173 (1981), URL http://www.sciencedirect.com/science/article/pii/05503213819

25 P. H. Ginsparg and K. G. Wilson, Physical Review D 25, 2649 (1982), URL http://link.aps.org/doi/10.1103/PhysRevD.25.2649.

26 M. Lüscher, Physics Letters B 428, 342 (1998), URL http://www.sciencedirect.com/science/article/pii/S0370269398

27 Y. Kikukawa and A. Yamada, Physics Letters B 448, $265 \quad$ (1999), URL http://www.sciencedirect.com/science/article/pii/S0370269399

28 H. Suzuki, Progress of Theoretical Physics 102, 141 (1999), http://ptp.oxfordjournals.org/content/102/1/141.full.pdf +html, URL http: //ptp. oxfordjournals .org/content/102/1/141. abstract

29 H. Neuberger, Physics Letters B 417, 141 (1998), URL

http://www.sciencedirect.com/science/article/pii/S0370269397

30 H. Neuberger, Physics Letters B 427, 353 (1998), URL http://www.sciencedirect.com/science/article/pii/S0370269398

31 D. H. Adams, Annals of Physics 296, 131 (2002), URL http://www.sciencedirect.com/science/article/pii/S0003491601

32 M. Lüscher, Nuclear Physics B 538, 515 (1999).

33 D. J. Thouless, M. Kohmoto, M. P. Nightingale, and M. den Nijs, Physical Review Letters 49, 405 (1982), URL bstrhthtp://link.aps.org/doi/10.1103/PhysRevLett.49.405. ${ }_{34}$ M. Kohmoto, Annals of Physics 160, 343 (1985).

35 T. Fukui, Y. Hatsugai, and H. Suzuki, Journal of the Physical Society of Japan 74, 1674 (2005), URL http://dx.doi.org/10.1143/JPSJ.74.1674. 